\title{
NORM INEQUALITIES FOR SOME ORTHOGONAL SERIES
}

\author{
BY RICHARD ASKEY ${ }^{1}$
}

To Professor A. Zygmund

1. Introduction. Over forty years ago M. Riesz [31] proved a theorem that is still inspiring new work. We refer to his celebrated conjugate function theorem.

Let $f\left(e^{i \theta}\right)$ be integrable on $(0,2 \pi)$ and form its Fourier series

$$
f\left(e^{i \theta}\right) \sim \sum_{-\infty}^{\infty} c_{n} e^{i n \theta}
$$

The conjugate function $\tilde{f}\left(e^{i \theta}\right)$ to $f\left(e^{i \theta}\right)$ is the function which in some sense has the expansion

$$
f\left(e^{i \theta}\right) \sim-i \sum_{-\infty}^{\infty}(\operatorname{sgn} n) c_{n} e^{i n \theta} .
$$

M. Riesz showed that if $f \in L^{p}, 1<p<\infty$, then $\tilde{f} \in L^{p}$, so (2) is an ordinary Fourier series, and

$$
\|\tilde{f}\|_{p} \leqq A_{p}\|f\|_{p}
$$

where

$$
\|f\|_{p}=\left[\int_{0}^{2 \pi}\left|f\left(e^{i \theta}\right)\right|^{p} d \theta\right]^{1 / p} .
$$

One reason for considering the conjugate function is that $f\left(e^{i \theta}\right)+i \tilde{f}\left(e^{i \theta}\right)$ has an analytic extension to the interior of the unit circle. In addition to this, there were two other applications of this theorem that were made many years ago. The first actually dates back before this theorem to a result of Kolmogoroff. Kolmogoroff [23] showed that the partial sums of (1) could be obtained in terms of $f$ and $\tilde{f}$. Using this M. Riesz proved the inequality

$$
\left\|S_{n}\right\|_{p} \leqq A_{p}\|f\|_{p} \quad(n=0,1, \cdots)
$$

where $S_{n}(f)=\sum_{-n}^{n} c_{k} e^{i k \theta}$ and $A_{p}$ is independent of $f$ and $n$. From (4) it was easily shown that $\lim _{n \rightarrow \infty}\left\|S_{n}-f\right\|_{p}=0$ for all $f \in L^{p}, 1<p<\infty$.

An address delivered by Professor Askey entitled Norm inequalities for some orthogonal expansions by invitation of the Committee to Select Hour Speakers for the Annual and Summer Meetings, on January 26, 1966; received by the editors April 8, 1966.

${ }^{1}$ Supported in part by N.S.F. grant GP-3483. 
A second application is due to Zygmund in the first edition of [38]. Hardy and Littlewood [17] proved that if

$$
f(x)=\sum_{n=1}^{\infty} a_{n} \cos n x
$$

and $a_{n+1} \leqq a_{n}, a_{n} \rightarrow 0$, then $f \in L^{p}$ if and only if $\sum a_{n}^{p} n^{p-2}$ converges, $1<p<\infty$. Since the conjugate function to $f(x)$ is the function with the expansion $\sum_{n=1}^{\infty} a_{n} \sin n x$, Zygmund observed that the theorem also holds for sine series, that is, if $g(x)=\sum_{n=1}^{\infty} a_{n} \sin n x, a_{n}$ as above, then $g \in L^{p}$ if and only if $\sum a_{n}^{p} n^{p-2}$ converges. In this case it is easy to dispense with the conjugate function theorem since the theorem for sine series can be proven in the same way as the theorem for cosine series. However the idea that one can get results for a series expansion from corresponding results for a different series expansion is an important idea as we will show later.

We now show how these three ideas can be applied in other contexts. We mention in detail only applications to orthogonal series. There are corresponding applications to Hankel transforms and to Dini series and we include references to these papers in the bibliography.

As the reader will see, many of the results I describe will be joint work with S. Wainger. In addition to the many mathematical discussions we have had for years, I discussed the organization of this paper with him and he made a number of valuable suggestions.

2. Mean convergence. Consider first the problem of mean convergence. The first important results for orthogonal expansions are due to Pollard [27], [28], [29], [30]. While his results have been proven for more general orthogonal expansions we state them only for ultraspherical expansions. The ultraspherical polynomials $P_{n}^{\lambda}(x)$ are defined by

$$
\left(1-2 r x+r^{2}\right)^{-\lambda}=\sum_{n=0}^{\infty} P_{n}^{\lambda}(x) r^{n} \quad \lambda>0 .
$$

They are orthogonal on $(-1,1)$ with respect to $\left(1-x^{2}\right)^{\lambda-1 / 2} d x$. We assume $f \in L^{p, \lambda}$; i.e., $N_{p}^{\lambda}[f]=\left[\int_{-1}^{1}|f(x)|^{p}\left(1-x^{2}\right)^{\lambda-1 / 2} d x\right]^{1 / p}$ is finite. Form its ultraspherical expansion

$$
f(x) \sim \sum_{n=0}^{\infty} a_{n}^{\lambda} P_{n}(x)
$$

where 


$$
a_{n}=\frac{2^{2 \lambda-1}[\Gamma(\lambda)]^{2}(n+\lambda) \Gamma(n+1)}{\pi \Gamma(n+2 \lambda)} \int_{-1}^{1} f(x) P_{n}^{\lambda}(x)\left(1-x^{2}\right)^{\lambda-1 / 2} d x .
$$

Let $S_{N} f=\sum_{n=0}^{N} a_{n} P_{n}^{\lambda}(x)$. Then Pollard [29] has shown that

$$
N_{p}^{\lambda}\left[S_{N} f\right] \leqq A_{p} N_{p}^{\lambda}[f] \quad(N=0,1, \cdots)
$$

but only for $(2 \lambda+1) /(\lambda+1)<p<(2 \lambda+1) / \lambda$. As usual from (5) we get $\lim _{N \rightarrow \infty} N_{p}^{\lambda}\left[S_{N} f-f\right]=0$.

Pollard has also shown that if

$$
f(x) \sim \sum b_{n} P_{n}^{\lambda}(x)\left(1-x^{2}\right)^{\lambda / 2-1 / 4}
$$

i.e.,

$$
b_{n}=\frac{2^{2 \lambda-1}[\Gamma(\lambda)]^{2}(n+\lambda) \Gamma(n+1)}{\pi \Gamma(n+2 \lambda)} \int_{-1}^{1} f(x) P_{n}^{\lambda}(x)\left(1-x^{2}\right)^{\lambda / 2-1 / 4} d x
$$

then

$$
\int_{-1}^{1}\left|\sum_{n=0}^{N} b_{n} P_{n}^{\lambda}(x)\left(1-x^{2}\right)^{\lambda / 2-1 / 4}-f(x)\right|^{p} d x \rightarrow 0 \text { for } 4 / 3<p<4
$$

when $f \in L^{p}, 4 / 3<p<4$.

The second result is not as interesting as the first, for among other reasons series of the form $\sum a_{n} P_{n}^{\lambda}(x)$ arise when considering expansions on spheres of zonal functions in terms of spherical harmonics. However, the fact that the indices $4 / 3$ and 4 are fixed turns out to be important. If we consider $\left(1-x^{2} / \lambda\right)^{\lambda-1 / 2}$ and let $\lambda \rightarrow \infty$ we get $\exp \left(-x^{2}\right)$. Also

$$
\lim _{\lambda \rightarrow \infty} \lambda^{-n / 2} P_{n}^{\lambda}\left(\frac{x}{\lambda^{1 / 2}}\right)=\frac{1}{n !} H_{n}(x)
$$

where $H_{n}(x)$ are the Hermite polynomials defined by $H_{n}(x)$ $=(-1)^{n} \exp \left(x^{2}\right)\left(d^{n} / d x^{n}\right)\left(\exp \left(-x^{2}\right)\right)$. See [35, p. 196.] These polynomials are orthogonal on $(-\infty, \infty)$ with respect to $\exp \left(-x^{2}\right) d x$. There are two interesting expansions which arise:

$$
f(x) \sim \sum a_{n} H_{n}(x)
$$

and

$$
f(x) \sim \sum b_{n} H_{n}(x) \exp \left(-x^{2}\right)
$$

where 


$$
\begin{aligned}
& a_{n}=\frac{1}{\pi^{1 / 2} 2^{n} n !} \int_{-\infty}^{\infty} f(x) H_{n}(x) \exp \left(-x^{2} / 2\right) d x \\
& b_{n}=\frac{1}{\pi^{1 / 2} 2^{n} n !} \int_{-\infty}^{\infty} f(x) H_{n}(x) \exp \left(-x^{2} / 2\right) d x
\end{aligned}
$$

It is of interest to see when the partial sums of (6) or (7) converge to $f$ in the appropriate mean. Since

$$
\lim _{\lambda \rightarrow \infty} \frac{2 \lambda+1}{\lambda+1}=\lim _{\lambda \rightarrow \infty} \frac{2 \lambda+1}{\lambda}=2
$$

we suspect that $\int_{-\infty}^{\infty}\left|\sum_{n=0}^{N} a_{n} H_{n}(x)-f(x)\right|^{p} \exp \left(-x^{2}\right) d x \rightarrow 0$ for all $f$ with $\int_{-\infty}^{\infty}|f(x)|^{p} \exp \left(-x^{2}\right) d x$ finite only if $p=2$. Pollard [29] has shown that this is correct. However the second result of Pollard held for $4 / 3<p<4$ so one might suspect that

$$
\int_{-\infty}^{\infty}\left|\sum_{n=0}^{N} b_{n} H_{n}(x) \exp \left(-x^{2} / 2\right)-f(x)\right|^{p} d x \rightarrow 0
$$

for all $f$ with $\int_{-\infty}^{\infty}|f(x)|^{p} d x$ finite, $4 / 3<p<4$. This result is true and was proven by Askey and Wainger in [2]. There we also proved the corresponding result for Laguerre series. Our proof uses an idea of Pollard to reduce the partial sum to a form with $\mathfrak{S}_{n}(x)-\mathfrak{S}_{n+2}(x)$ instead of $\mathfrak{S}_{n}(x)-\mathfrak{S}_{n+1}(x)$, which is what naturally appears. For certain values of $x, \mathfrak{S}_{n}(x)-\mathfrak{S}_{n+2}(x)$ is smaller than $\mathfrak{S}_{n}(x)-\mathfrak{S}_{n+1}(x)$. $\mathfrak{S}_{n}(x)$ is the orthonormal Hermite function. We also use important asymptotic formulas of Erdélyi and Skovgaard [12]. We should remark that all of these results are best possible in that they fail for some $f \in L^{p}$ for $p$ either endpoint of the appropriate open interval.

Pollard has asked the question about how you guess the values of $p$ for which there is mean convergence for the expansion in orthogonal polynomials which are orthogonal with respect to $w(x) d x$. This problem seems to be related to another problem on orthogonal series, the problem of what Cèsaro means sum a series in $L^{1}$. For ultrapsherical series, $\sum_{n=0}^{\infty} a_{n} P_{n}^{\lambda}(x)$, the $(C, \lambda+\epsilon)$ means are effective in $L^{1}$, i.e.

$$
\int_{-1}^{1}\left|\frac{1}{A_{N}^{\lambda+\epsilon}} \sum_{n=0}^{N} A_{N-n}^{\lambda+\epsilon} a_{n} P_{n}^{\lambda}(x)-f(x)\right|\left(1-x^{2}\right)^{\lambda-1 / 2} d x \rightarrow 0
$$

for all $f \in L^{1}\left(\left(1-x^{2}\right)^{\lambda-1 / 2} d x\right)$. Here

$$
A_{N}^{\alpha}=\left(\begin{array}{c}
N+\alpha \\
N
\end{array}\right)
$$


On a $(1 / p, \alpha)$ plane plot the points $((\lambda+1) /(2 \lambda+1), 0)$ and $(1, \lambda)$.

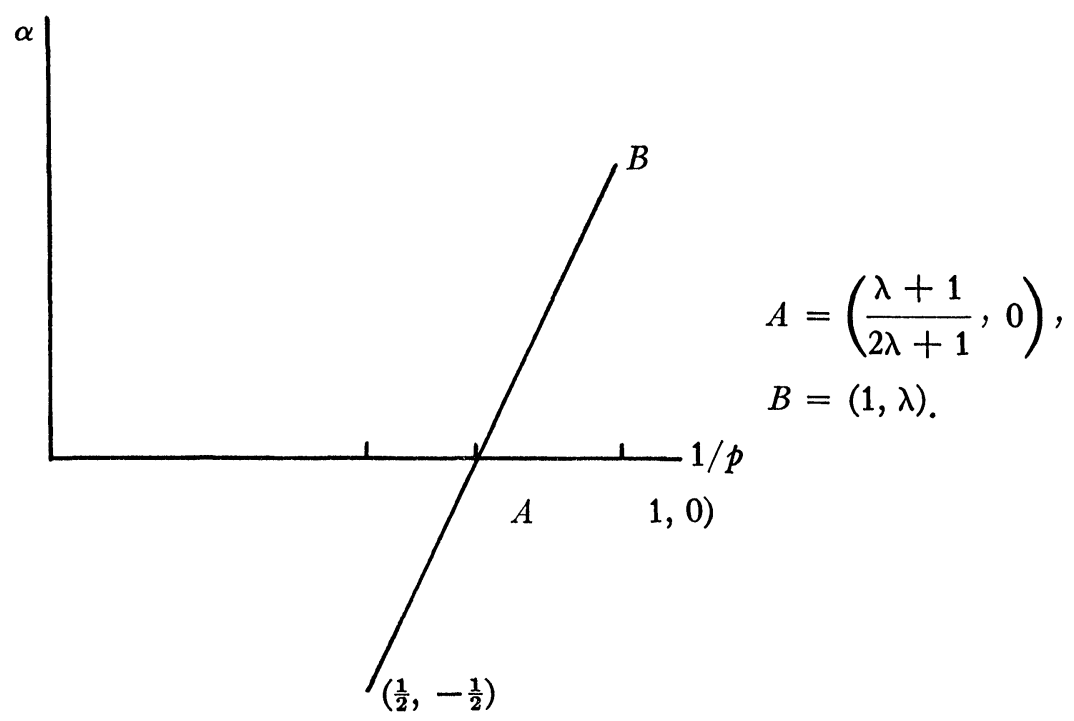

If we connect them with a straight line we see that the point $\left(\frac{1}{2},-\frac{1}{2}\right)$ is collinear with them. Whether this is always true is unknown for there are few cases in which both the mean convergence and the mean summability problems have been solved. Getting away from orthogonal polynomials, but using this principle, it is natural to conjecture that for spherical partial sums of multiple Fourier series and for expansions in spherical harmonics that mean convergence holds in $L^{p}$ for $2 n /(n+1)<p<2 n /(n-1)$ where $n$ is the dimension of the space we are in. For each of these problems the corresponding $(C, \alpha)$ summability problem has been solved, [10], [22], [32].

A somewhat more tractable conjecture is obtained if we consider the problem of Cèsaro summability for Jacobi or Hermite series. Jacobi polynomials, $P_{n}^{(\alpha, \beta)}(x)$, are polynomials orthogonal on $(-1,1)$ with respect to $(1-x)^{\alpha}(1+x)^{\beta}$. For these polynomials the mean convergence problem has been solved, [27], [30], but the mean Cèsaro summability in $L^{1}$ is still open, [1]. It should be possible to find an analogue of the convolution structure that is known for the case $\alpha=\beta$ and then the mean Cèsaro summability would follow from known results on the Lebesgue constants [1], [35]. And even without the convolution structure the theorem can probably be proven because of the known uniform asymptotic formulas of Hilb type [34], [35].

For Hermite series, $\sum a_{n} H_{n}(x)$, it was shown in [1] that no Cèsaro 
mean is effective in $L^{1}$, or even $L^{p}, p \neq 2$. For a closely related orthogonal set of functions, the Laguerre polynomials, D. Ernst [13] has shown that the $(C, 1)$ means are effective in $L^{1}$. More particularly, he shows that if $\int_{0}^{\infty}|f(x)| d x<\infty$ and

$$
f(x) \sim \sum a_{n} x^{\alpha / 2} L_{n}^{\alpha}(x) \exp (-x / 2)
$$

then the $(C, 1)$ means converge in $L^{1}$ norm. For technical reasons he assumes that $\alpha \geqq 3$ but this restriction is probably not necessary. If this theorem were known for $\alpha= \pm \frac{1}{2}$, then it would be known for Hermite series. Actually the best result is probably that the $\left(C, \frac{1}{2}+\epsilon\right)$ means are effective while the $\left(C, \frac{1}{2}\right)$ means are not.

For another heuristic connection between Cèsaro summability and convergence see [24]. For related results on Bessel series and integrals see $[16],[19],[36],[37]$.

3. Mapping theorems. We now take up another of the ideas mentioned in the introduction, the problem of getting results for expansions in a family of orthogonal functions from known results on expansions in other orthogonal functions. We start by recalling Parsevall's theorem.

Let $f(\theta) \in L^{2}(0, \pi)$ and define its Fourier coefficients by $a_{n}=(2 / \pi)$ $\int_{0}^{\pi} f(\theta) \cos n \theta d \theta$. Then $\sum\left|a_{n}\right|^{2}$ is finite and

$$
\frac{a_{0}^{2}}{2}+\sum_{n=1}^{\infty} a_{n}^{2}=\frac{2}{\pi} \int_{0}^{\pi}|f(\theta)|^{2} d \theta .
$$

Let $P_{n}^{\lambda}(\cos \theta)$ be the ultraspherical polynomials of degree $n$, order $\lambda$, $\lambda>0$, and define two functions $f^{\lambda}$ and $f_{\lambda}$ by

$$
f^{\lambda}(\theta)=\sum a_{n} \hat{t}_{n}^{\lambda} P_{n}^{\lambda}(\cos \theta)(\sin \theta)^{\lambda}
$$

and

$$
f_{\lambda}(\theta)=\sum a_{n} t_{n}^{\lambda} P_{n}^{\lambda}(\cos \theta) .
$$

Here

$$
\hat{t}_{n}^{\lambda}=\left[\frac{2^{2 \lambda-1}[\Gamma(\lambda)]^{2}(n+\lambda) \Gamma(n+1)}{\pi \Gamma(n+2 \lambda)}\right]^{1 / 2}
$$

and

$$
\int_{0}^{\pi}\left(l_{n}^{\lambda}\right)^{2}\left[P_{n}^{\lambda}(\cos \theta)\right]^{2}(\sin \theta)^{2 \lambda} d \theta=1
$$


Since $\sum\left|a_{n}\right|^{2}$ is finite each of the series (9) and (10) converges in an appropriate $L^{2}$ space and we have

$$
\int_{0}^{\pi}\left|f^{\lambda}(\theta)\right|^{2} d \theta=\sum_{n=0}^{\infty}\left|a_{n}\right|^{2}
$$

and

$$
\int_{0}^{\pi}\left|f_{\lambda}(\theta)\right|^{2}(\sin \theta)^{2 \lambda} d \theta=\sum_{n=0}^{\infty}\left|a_{n}\right|^{2}
$$

It is clear from (8), (11), and (12) that

$$
\int_{0}^{\pi}|f(\theta)|^{2} d \theta \sim \int_{0}^{\pi}\left|f^{\lambda}(\theta)\right|^{2} d \theta
$$

and

$$
\int_{0}^{\pi}|f(\theta)|^{2} d \theta \sim \int_{0}^{\pi}\left|f_{\lambda}(\theta)\right|^{2}(\sin \theta)^{2 \lambda} d \theta .
$$

We write $a \sim b$ if $a / b \leqq C$ and $b / a \leqq C$ where $C$ is a constant (which is independent of any free variables in $a$ or $b$ ). For instance in (13), $\lambda$ is fixed but $f$ is free.

We would like to generalize (13) or (14) to values of $p$ other than $p=2$. For instance we would like to see for what $p$ we have

$$
\int_{0}^{\pi}|f(\theta)|^{p} d \theta \sim \int_{0}^{\pi}\left|f^{\lambda}(\theta)\right|^{p} d \theta
$$

and for what $p$ we have

$$
\int_{0}^{\pi}|f(\theta)|^{p} d \theta \sim \int_{0}^{\pi}\left|f_{\lambda}(\theta)\right|^{p}(\sin \theta)^{2 \lambda} d \theta .
$$

(16) is false for $p \leqq(2 \lambda+1) /(\lambda+1)$ and $p \geqq(2 \lambda+1) / \lambda$ since we have mean convergence of Fourier series for $1<p<\infty$ but mean convergence of ultraspherical series only for $(2 \lambda+1) /(\lambda+1)<p<(2 \lambda+1) / \lambda$. Actually (16) is false for all $p \neq 2$ as Wainger and I showed in [4]. We will give this example later in this section.

On the other hand (15) is true for $1<p<\infty$ and it is also true in certain weighted norms as was shown in [5]. There are a couple of reasons why a result like (15) is to be expected. From the known asymptotic relations for $P_{n}^{\lambda}(\cos \theta)$ we have

$$
{ }_{t_{n}}^{\lambda} P_{n}^{\lambda}(\cos \theta)(\sin \theta)^{\lambda}=A \cos [(n+\lambda) \theta-\lambda \pi / 2]+O(n \sin \theta)^{-1} .
$$

Thus the series $\sum a_{n} t_{n}^{\lambda} P_{n}^{\lambda}(\cos \theta)(\sin \theta)^{\lambda}$ is actually $\sum\left[a_{n} \cos [(n+\lambda) \theta\right.$ $\left.-\lambda \pi / 4]+O\left(a_{n} /(n \sin \theta)\right)\right]$ and for $\theta$ bounded away from 0 and $\pi$ 
the series is essentially $\sum a_{n} \cos [(n+\lambda) \theta-\lambda \pi / 2]$. By M. Riesz's theorem this series behaves like $\sum a_{n} \cos n \theta$, at least as far as its integrability properties are concerned. This connection has been used for many decades to set up equiconvergence theorems between Fourier and ultraspherical series on compact subsets on $(0, \pi)$. However, no one has succeeded in using only the asymptotic formulas to get connections on all of $[0, \pi]$. After formally stating our theorem we will give the second heuristic reason and it is this reason that has been turned into a proof.

Theorem 1. Let $1<p<\infty,-1<\alpha<p-1$. Let $\int_{0}^{\pi}|f(\theta)|^{p}(\sin \theta)^{\alpha} d \theta$ $<\infty$. Define

so that

$$
a_{n}=\frac{2}{\pi} \int_{0}^{\pi} f(\theta) \cos n \theta d \theta
$$

Define

$$
f(\theta) \sim \sum a_{n} \cos n \theta .
$$

$$
T_{r}^{\lambda} f(\theta)=f_{r}^{\lambda}(\theta)=\sum_{n=0}^{\infty} r^{n} a_{n} t_{n}^{\lambda} P_{n}^{\lambda}(\cos \theta)(\sin \theta)^{\lambda}, \quad 0<r<1 .
$$

Then

$$
\left[\int_{0}^{\pi}\left|f_{r}^{\lambda}(\theta)\right|^{p}(\sin \theta)^{\alpha} d \theta\right]^{1 / p} \leqq A\left[\int_{0}^{\pi}|f(\theta)|^{p}(\sin \theta){ }^{\alpha} d \theta\right]^{1 / p}
$$

where $A$ is independent of $r$ and $f$. Also there is $f^{\lambda}(\theta)$ such that $f_{r}^{\lambda}(\theta)$ $\rightarrow f^{\lambda}(\theta)$ a.e. as $r \rightarrow 1$. Finally

and

$$
\left[\int_{0}^{\pi}\left|f_{r}^{\lambda}(\theta)-f^{\lambda}(\theta)\right|^{p}(\sin \theta)^{\alpha} d \theta\right]^{1 / p} \rightarrow 0
$$

$$
\left[\int_{0}^{\pi}\left|f^{\lambda}(\theta)\right|^{p}(\sin \theta)^{\alpha} d \theta\right]^{1 / p} \leqq A\left[\int_{0}^{\pi}|f(\theta)|^{p}(\sin \theta)^{\alpha} d \theta\right]^{1 / p}
$$

There is also a related theorem in which one expands first in $t_{n}^{\lambda} P_{n}^{\lambda}(\cos \theta)(\sin \theta)^{\lambda}$ and uses these coefficients to set up a new function defined by a cosine series and then the same inequalities hold.

Theorem 2. Let $p, \alpha, f$ be as in Theorem 1. Define

so that

$$
b_{n}=\int_{0}^{\pi} f(\theta) t_{n}^{\lambda} P_{n}^{\lambda}(\cos \theta)(\sin \theta)^{\lambda} d \theta
$$

$$
f(\theta) \sim \sum b_{n} t_{n}^{\lambda} P_{n}^{\lambda}(\cos \theta)(\sin \theta)^{\lambda} .
$$


Set

$$
S_{r}^{\lambda} f(\theta)=\sum_{n=0}^{\infty} b_{n} r^{n} \cos n \theta
$$

Then

$$
\left[\int_{0}^{\pi}\left|S_{r}^{\lambda} f(\theta)\right|^{p}(\sin \theta)^{\alpha} d \theta\right]^{1 / p} \leqq A\left[\int_{0}^{\pi}|f(\theta)|^{p}(\sin \theta)^{\alpha} d \theta\right]^{1 / p}
$$

where $A$ is independent of $r$ and $f$. Also there is $S^{\lambda} f(\theta)$ such that $S_{r}^{\lambda} f(\theta)$ $\rightarrow S^{\lambda} f(\theta)$ a.e., $\left[\int_{0}^{\pi}\left|S_{r}^{\lambda} f(\theta)-S^{\lambda} f(\theta)\right|^{p}(\sin \theta)^{\alpha} d \theta\right]^{1 / p} \rightarrow 0$, and

$$
\left[\int_{0}^{\pi}\left|S^{\lambda} f(\theta)\right|^{p}(\sin \theta)^{\alpha} d \theta\right]^{1 / p} \leqq A\left[\int_{0}^{\pi}|f(\theta)|^{p}(\sin \theta)^{\alpha} d \theta\right]^{1 / p} .
$$

For $\lambda=1 / 2$ we sketch the idea of the proof. Recall Mehler's formula

$$
P_{n}^{1 / 2}(\cos \theta)=\pi^{-1} 2^{1 / 2} \int_{0}^{\theta} \frac{\cos \left(n+\frac{1}{2}\right) \phi}{(\cos \phi-\cos \theta)^{1 / 2}} d \phi .
$$

Using this in $\sum a_{n} r^{n}(n+1 / 2)^{1 / 2} P_{n}^{(1 / 2)}(\cos \theta)(\sin \theta)^{1 / 2}$ we see that

$$
T_{r}^{\lambda} f(\theta)=2^{1 / 2} \pi^{-1} \int_{0}^{\theta} \frac{\sum a_{n} r^{n}\left(n+\frac{1}{2}\right)^{1 / 2} \cos \left(n+\frac{1}{2}\right) \phi}{[\cos \phi-\cos \theta]^{1 / 2}} d \phi(\sin \theta)^{1 / 2} .
$$

The series is related to the fractional derivative of order one half of $\sum a_{n} r^{n} \cos n \phi$ and the integral is related to the fractional integral of order one half. The proof consists of unscrambling these two operations.

Before we give applications of these theorems we need to state some results about Fourier series. We state them only for cosine series, but they in fact hold for general Fourier series. The first theorem is a generalization of the Marcinkiewicz multiplier theorem to weighted $L^{p}$ spaces and is due to Hirschman [20].

Theorem A. Let $\int_{0}^{\pi}|f(\theta)|^{p}(\sin \theta)^{\alpha} d \theta<\infty, 1<p<\infty,-1<\alpha<p-1$. Set

$$
a_{n}=\frac{2}{\pi} \int_{0}^{\pi} f(\theta) \cos n \theta d \theta
$$

so

$$
f(\theta) \sim \sum a_{n} \cos n \theta .
$$


If $\left\{t_{n}\right\}$ satisfies

$$
\begin{gathered}
\left|t_{n}\right| \leqq C \quad n=0 \quad 1, \cdots \\
\sum_{2^{n}}^{2^{n+1}-1}\left|t_{k+1}-t_{k}\right| \leqq C \quad n=0 \quad 1, \cdots
\end{gathered}
$$

then there is a function Tf such that

$$
T f(\theta) \sim \sum t_{n} a_{n} \cos n \theta
$$

and

$$
\left[\int_{0}^{\pi}|T f(\theta)|^{p}(\sin \theta)^{\alpha} d \theta\right]^{1 / p} \leqq A C\left[\int_{0}^{\pi}|f(\theta)|^{p}(\sin \theta)^{\alpha} d \theta\right]^{1 / p}
$$

where $A$ is independent of $f$ and $\left\{t_{n}\right\}$.

For $t_{k}=1$ if $n \leqq N$ and $t_{n}=0$ if $n>N$ this theorem is essentially due to Hardy and Littlewood [18] and for $\alpha=0$ it is the theorem of M. Riesz that we mentioned in the introduction.

A second theorem is due to Hardy and Littlewood [17] for monotonic coefficients and is found in [7] in this degree of generality.

Theorem B. Let $f$ be integrable on $(0, \pi)$. Then if

$$
a_{n}=\frac{2}{\pi} \int_{0}^{\pi} f(\theta) \cos n \theta d \theta
$$

and $(n+1)^{-k} a_{n+1} \leqq n^{-k} a_{n}$ for some $k$, we have $\int_{0}^{\pi}|f(\theta)|^{p}(\sin \theta)^{\alpha} d \theta<\infty$ if and only if $\sum a_{n}^{p} n^{p-2-\alpha}<\infty, 1 \leqq p<\infty,-1<\alpha<p-1$.

An analogue of the Marcinkiewicz theorem is the following.

TheOREM 3. Let $\int_{0}^{\pi}|f(\theta)|^{p}(\sin \theta)^{2 \lambda} d \theta<\infty, \quad(2 \lambda+1) /(\lambda+1)<p$ $<(2 \lambda+1) / \lambda, f(\theta) \sim \sum a_{n} P_{n}^{\lambda}(\cos \theta)$. Assume that $\left|s_{N}\right| \leqq C$ and $\sum_{2^{N}}^{2^{N+1}}\left|s_{n}-s_{n+1}\right| \leqq C, N=0,1,2, \cdots$. Then there is $T f(\theta)$ such that

$$
T f(\theta) \sim \sum a_{n} s_{n} P_{n}^{\lambda}(\cos \theta)
$$

and

$$
\int_{0}^{\pi}|T f(\theta)|^{p}(\sin \theta)^{2 \lambda} d \theta \leqq A \int_{0}^{\pi}|f(\theta)|^{p}(\sin \theta)^{2 \lambda} d \theta
$$

Using Theorems 1,2 , and $A$ we see that 


$$
\begin{aligned}
\int_{0}^{\pi}|T f(\theta)|^{p}(\sin \theta)^{2 \lambda} d \theta & \\
& =\int_{0}^{\pi}\left|\sum a_{n} s_{n} P_{n}^{\lambda}(\cos \theta)(\sin \theta)^{\lambda}\right|^{p}(\sin \theta)^{2 \lambda-p \lambda} d \theta \\
& \leqq A \int_{0}^{\pi}\left|\sum\left(t_{n}\right)^{\lambda-1} a_{n} s_{n} \cos n \theta\right|^{p}(\sin \theta)^{2 \lambda-p \lambda} d \theta \\
& \leqq A \int_{0}^{\pi}\left|\sum\left(t_{n}\right)^{\lambda-1} a_{n} \cos n \theta\right|^{p}(\sin \theta)^{2 \lambda-p \lambda} d \theta \\
& \leqq A \int_{0}^{\pi}\left|\sum a_{n} P_{n}^{\lambda}(\cos \theta)(\sin \theta)^{\lambda}\right|^{p}(\sin \theta)^{2 \lambda-p \lambda} d \theta \\
& =A \int_{0}^{\pi}|f(\theta)| p(\sin \theta)^{2 \lambda} d \theta .
\end{aligned}
$$

We need $-1<2 \lambda-p \lambda<p-1$ or $(2 \lambda+1) /(\lambda+1)<p<(2 \lambda+1) / \lambda$.

Similarly the following analogue of the Hardy-Littlewood theorem holds.

TheOREM 4. Let $f(\theta) \sim \sum a_{n} P_{n}^{\lambda}(\cos \theta)$ where $n^{-k} a_{n} \downarrow 0$ for some $k$. Then $\int_{0}^{\pi}|f(\theta)|^{p}(\sin \theta)^{\alpha}(\sin \theta)^{2 \lambda} d \theta<\infty$ if and only if $\sum\left|a_{n}\right|^{p} n^{2 p-2-2 \lambda-\alpha}$ $<\infty, 1<p<\infty, \lambda p-(1+2 \lambda)<\alpha<p(\lambda+1)-(1+2 \lambda)$.

There is a related $L^{1}$ result but only for series with monotone coefficients. As a corollary of other results, Ganser [14] shows that

$$
f(\theta) \sim \sum a_{n} \cos n \theta \quad f^{\lambda}(\theta) \sim \sum a_{n} t_{n}^{\lambda} P_{n}^{\lambda}(\cos \theta)(\sin \theta)^{\lambda}
$$

satisfy $\int_{0}^{\pi}|f(\theta)|(\sin \theta)^{\beta-1} d \theta<\infty$ if and only if $\int_{0}^{\pi}\left|f^{\lambda}(\theta)\right|(\sin \theta)^{\beta-1} d \theta<\infty$ but only for $0<\beta<1$ and more importantly if some condition like $a_{n+2} \leqq a_{n}, a_{n} \rightarrow 0$ holds. It would be interesting to have some counterexamples to this result without the assumption on $a_{n}$.

To show that the mapping between $\sum a_{n} \cos n \theta$ and $\sum a_{n} t_{n}^{\lambda} P_{n}^{\lambda}(\cos \theta)$ is not bounded we consider the functions given by $f(\theta)=\sum n^{-\alpha} \cos n \theta$ and $g(\theta)=\sum n^{-\alpha} t_{n}^{\lambda} P_{n}^{\lambda}(\cos \theta)$ and the functions given by $T f(\theta)$ $=\sum i^{n} n^{-\alpha} \cos n \theta$ and $T g(\theta)=\sum i^{n} n^{-\alpha} t_{n}^{\lambda} P_{n}^{\lambda}(\cos \theta)$ for $0<\alpha<1$. We have that $f(\theta)$ and $g(\theta)$ are continuous except for $\theta=0$ and there $f(\theta)=A \theta^{\alpha-1}+B(\theta), g(\theta)=A^{\prime} \theta^{\alpha-\lambda-1}+B^{\prime}(\theta)$, where $A$ and $A^{\prime}$ are constants, $B(\theta)$ is bounded and $B^{\prime}(\theta)=O\left(\theta^{\alpha-\lambda}+1\right)$. See [3, p. 215]. Tf( $\left.\theta\right)$ and $\operatorname{Tg}(\theta)$ are continuous except for $\theta=\pi / 2$ and there

$$
\begin{aligned}
& T f(\theta) \sim|\theta-\pi / 2|^{\alpha-1}\left[A_{1}+B_{1} \operatorname{sgn}(\theta-\pi / 2)\right], \\
& T g(\theta) \sim|\theta-\pi / 2|^{\alpha-1}\left[A_{2}+B_{2} \operatorname{sgn}(\theta-\pi / 2)\right]
\end{aligned}
$$


where $A_{1}, B_{1}, A_{2}, B_{2}$ are constants and at least one each of $A_{1}, B_{1}$ and $A_{2}, B_{2}$ is nonzero. See [4]. $f(\theta)$ and $T f(\theta)$ are in $L^{p}(d \theta)$ for the same values of $\alpha$. However $g \in L^{p}\left((\sin \theta)^{2 \lambda} d \theta\right)$ for $\alpha>1+\lambda-(1+2 \lambda) / p$ and $T g \in\left((\sin \theta)^{2 \lambda} d \theta\right)$ for $\alpha>1-1 / p$ but not for other values of $\alpha$.

Thus if $p<2$ and $1+\lambda-(1+2 \lambda) / p<\alpha<1-1 / p$, we have $g \in L^{p}\left((\sin \theta)^{2 \lambda} d \theta\right)$ but not $T g$. However $f$ and $T f$ belong to $L^{p}$ at the same time and so it is not possible to set up a mapping between $\sum a_{n} \cos n \theta$ and $\sum a_{n} t_{n}^{\lambda} P_{n}^{\lambda}(\cos \theta)$ that is bounded in both directions. Actually this shows slightly more, that the only way we could set up a mapping between $\sum a_{n} \cos n \theta$ and $\sum a_{n} k_{n} P_{n}^{\lambda}(\cos \theta)(\sin \theta)^{\beta}$ and have it bounded in both directions is to have $k_{n} \sim t_{n}^{\lambda}$ and $\beta=\lambda$ and have the measures the same.

There is a dual mapping theorem in which the function $f$ is held fixed and the mapping goes between different coefficients.

Theorem 5. Let $f(\theta) \in L^{1}(0, \pi)$ and $1<p<\infty,-1<\alpha<p-1$. Set

$$
a_{n}=\int_{0}^{\pi} f(\theta) \cos n \theta d \theta
$$

and

$$
a_{n}^{\lambda}=\int_{0}^{\pi} f(\theta) t_{n}^{\lambda} P_{n}^{\lambda}(\cos \theta)(\sin \theta)^{\lambda} d \theta, \quad \lambda>0 .
$$

Then if either $\sum\left|a_{n}\right|^{p}(n+1)^{\alpha}<\infty$ or $\sum\left|a_{n}^{\lambda}\right|^{p}(n+1)^{\alpha}<\infty$ so is the other and

$$
\left[\sum_{n=0}^{\infty}\left|a_{n}\right|^{p}(n+1)^{\alpha}\right]^{1 / p} \sim\left[\sum_{n=0}^{\infty}\left|a_{n}^{\lambda}\right|^{p}(n+1)^{\alpha}\right]^{1 / p} .
$$

This theorem is in [6]. There is a corresponding theorem for Jacobi coefficients [8] and in this paper there are some results on the mapping in $l^{1}$. The applications that follow from Theorem 5 are handled in exactly the same way as the applications that were given following Theorems 1 and 2. The analogue of the Marcinkiewicz theorem is due to Sunonchi [33] and Igari [21].

For a similar theorem for Hankel transforms see [16]. There are also two other papers that have work that is related to this type of theorem [9], [15]. Baxter's work is related to Theorem 5 and Gunzler's work is related to Theorems 1 and 2. However, they are both different in that the mappings they set up are bounded in $L^{1}$ (or $l^{1}$ ) while our mappings are not.

4. Conjugate series. Finally we mention briefly a few of the results of Muckenhoupt and Stein [25]. These results are closely related to 
our Theorems 1 and 2. In fact we prove Theorem 1 first for $0<\lambda<1$ and then use estimates found by Muckenhoupt and Stein to extend our result to $\lambda \geqq 1$.

We have considered expansions

$$
f(\theta) \sim \sum a_{n} P_{n}^{\lambda}(\cos \theta)
$$

in both $\S \S 2$ and 3. To this function $f(\theta)$ we associate the "harmonic" function

$$
f(r, \theta)=\sum a_{n} r^{n} P_{n}^{\lambda}(\cos \theta) \quad 0 \leqq r<1 .
$$

If we write $u(x, y)=f(r, \theta), x=r \cos \theta, y=r \sin \theta$, then $u(x, y)$ satisfies

$$
\Delta_{\lambda}(u)=\frac{\partial^{2} u}{\partial x^{2}}+\frac{\partial^{2} u}{\partial y^{2}}+\frac{2 \lambda}{y} \frac{\partial u}{\partial y}=0
$$

for $x^{2}+y^{2}<1, y>0$. To the series for $f(\theta)$ we associate a "conjugate" series

$$
f(\theta) \sim 2 \lambda \sum_{n=1}^{\infty} \frac{a_{n}}{n+2 \lambda} P_{n-1}^{\lambda+1}(\cos \theta) \sin \theta
$$

and its "conjugate harmonic" series

$$
\tilde{f}(r, \theta)=2 \lambda \sum_{n=1}^{\infty} \frac{a_{n}}{n+2 \lambda} r^{n} P_{n-1}^{\lambda+1}(\cos \theta) \sin \theta .
$$

If we set

$$
v(x, y)=y^{2 \lambda} \tilde{f}(r, \theta)
$$

then $u$ and $v$ satisfy "Cauchy-Riemann equations"

$$
v_{x}=-y^{2 \lambda} u_{y}, \quad v_{y}=y^{2 \lambda} u_{x}
$$

and $v$ satisfies an equation "conjugate" to that of $u$,

$$
\Delta_{-\lambda}(v)=\frac{\partial^{2} v}{\partial x^{2}}+\frac{\partial^{2} v}{\partial y^{2}}-\frac{2 \lambda}{y} \frac{\partial v}{\partial y}=0 .
$$

Since $P_{n}^{1}(\cos \theta)=\sin (n+1) \theta / \sin \theta$ and $\lim _{\lambda \rightarrow 0}((n+2 \lambda) / 2 \lambda) P_{n}^{\lambda}(\cos \theta)$ $=\cos n \theta$ the above reduce to the classical notion of conjugate function when $\lambda \rightarrow 0$.

In some sense $f+i \tilde{f}$ is a generalized analytic function. There are two types of theorems we now mention. The first is a generalization of M. Riesz's conjugate function theorem. Muckenhoupt and Stein show that 


$$
\left[\int_{0}^{\pi}|\tilde{f}(\theta)|^{p}(\sin \theta)^{2 \lambda} d \theta\right]^{1 / p} \leqq A\left[\int_{0}^{\pi}|f(\theta)|^{p}(\sin \theta)^{2 \lambda} d \theta\right]^{1 / p}
$$

for $1<p<\infty$ and conversely that

$$
\left[\int_{0}^{\pi}|f(\theta)|^{p}(\sin \theta)^{2 \lambda} d \theta\right]^{1 / p} \leqq A\left[\int_{0}^{\pi}|\tilde{f}(\theta)| p(\sin \theta)^{2 \lambda} d \theta\right]^{1 / p} .
$$

As we remarked above the analysis that went into the proof of this theorem was used to complete the proof of Theorem 1.

The other theorem is also an analogue of a classical theorem. If $f(z)=u(x, y)+i v(x, y)$ is analytic then $|f(z)|^{\alpha}=\left(u^{2}+v^{2}\right)^{\alpha / 2}$ is subharmonic for $\alpha>0$. The analogue of this for ultraspherical expansions is the following.

TheOREm 6. Let $f(r, \theta)$ and $\tilde{f}(r, \theta)$ be defined by (18) and (19). Then $\Delta_{\lambda}\left[f^{2}+\tilde{f}^{2}\right]^{p / 2} \geqq 0$ for $p \geqq 2 \lambda /(2 \lambda+1)$.

A corollary of this is the following theorem which is the analogue of the F. and M. Riesz theorem.

Theorem 7. Let $\int_{0}^{\pi}(\sin \theta)^{2 \lambda} d \mu<\infty, \int_{0}^{\pi}(\sin \theta)^{2 \lambda} d \nu<\infty$ and

and

$$
d \mu \sim \sum a_{n} P_{n}^{\lambda}(\cos \theta)
$$

$$
d \nu \sim 2 \lambda \sum \frac{a_{n}}{n+2 \lambda} P_{n-1}^{\lambda+1}(\cos \theta) \sin \theta .
$$

Then $d \mu$ and $d \nu$ are absolutely continuous.

There are many other theorems in this interesting paper including the first proof of the Marcinkiewicz multiplier theorem which we gave in $\$ 3$. The results in this paper are only for $\lambda>0$. For $-\frac{1}{2}<\lambda<0$ the ultraspherical series still make sense and a start of the theory for this case has been made by S. Parter in [26].

\section{BIBLIOGRAPHY}

1. R. Askey and I. I. Hirschman, Jr., Mean summability for ultraspherical polynomials, Math. Scand. 12 (1963), 167-177.

2. R. Askey and S. Wainger, Mean convergence of expansions in Laguerre and Hermite series, Amer. J. Math. 87 (1965), 695-708.

3. - On the behavior of special classes of ultraspherical expansions. I, J. Anal. Math. 15 (1965), 193-220.

4. - On the behavior of special classes of ultrapherical expansions. II, J. Anal. Math. 15 (1965), 221-244.

5. —, A transplantation theorem between ultraspherical series, Illinois J. Math. 10 (1966), 322-344. 
6. - A transplantation theorem for ultraspherical coefficients, Pacific J. Math. 16 (1966), 393-405.

7. - Integrability theorems for Fourier series, Duke Math. J. 33 (1966), 223-228.

8. R. Askey, A transplantation theorem for Jacobi coefficients, Pacific J. Math. (to appear).

9. G. Baxter, $A$ convergence equivalence related to polynomials orthogonal on the unit circle, Trans. Amer. Math. Soc. 99 (1961), 471-487.

10. K. K. Chen, On the Cèsaro-summability of the Laplace's series of hyperspherical functions, Sci. Rep. Tôhoku Imp. Univ. Ser. 1, 17 (1928), 1073-1089.

11. A. Erdélyi et. al., Bateman Manuscript Project, H.T.F. vol. 2, New York, 1953.

12. A. Erdélyi, Asymptotic solutions of differential equations with transition points or singularities, J. Math. Phys. 1 (1960), 16-26.

13. D. Ernst, Über die Laguerre-transformation und ihre Umkehrung, unpublished Doctoral Dissertation, Aachen, 1965.

14. C. Ganser, Integrability theorems for ultraspherical series, Duke Math. J, (to appear).

15. H. Gunzler, Verallgemeinerte Fourierreihen, Nachr. Akad. Wiss. Göttingen Math.-Phys. Kl. II 1962, 81-122.

16. D. Guy, Hankel multiplier transformations and weighted p-norms, Trans. Amer. Math Soc. 95 (1960), 137-189.

17. G. H. Hardy and J. E. Littlewood, Some new properties of Fourier coefficients, J. London Math. Soc. 6 (1931), 3-9.

18. - Some theorems on Fourier series and Fourier power series, Duke Math. J. 2 (1936), 354-381.

19. C. Herz, On the mean inversion of Fourier and Hankel transforms, Proc. Nat. Acad. Sci. U.S.A. 40 (1954), 996-999.

20. I. I. Hirschman, Jr., Decomposition of Walsh and Fourier series, Mem. Amer. Math. Soc., no. 15, 1955.

21. S. Igari, On the decomposition theorems of Fourier transforms with weighted norms, Tôhoku Math. J. 15 (1963), 6-36.

22. E. Kogbetliantz, Recherches sur la sommabilité des séries ultrasphêriques par la méthode des moyennes arithmétiques, J. Math. Pures Appl. (3) 9 (1924), 107-187.

23. A. Kolmogoroff, Sur les fonctions harmonique conjugées et les sếries de Fourier, Fund. Math. 7 (1925), 24-29.

24. L. Lorch, The Lebesque constant for Jacobi series. I, Proc. Amer. Math. Soc. 10 (1959), 756-761.

25. B. Muckenhoupt and E. M. Stein, Classical expansions and their relation to conjugate harmonic functions, Trans. Amer. Math. Soc. 118 (1965), 17-92.

26. S. Parter, On the existence and uniqueness of symmetric axially symmetric potentials, Arch. Rational Mech. Anal. 20 (1965), 279-286.

27. H. Pollard, The mean convergence of orthogonal series of polynomials, Proc. Nat. Acad. Sci. U.S.A. 32 (1946), 8-10.

28. - The mean convergence of orthogonal series. I, Trans. Amer. Math. Soc. 62 (1947), 387-403.

29. - The mean convergence of orthogonal series. II, Trans. Amer. Math. Soc. 63 (1948), 355-367.

30. - The mean convergence of orthogonal series. III, Duke Math. J. 16 (1949), 189-191.

31. M. Riesz, Sur les fonctions conjuguées, Math. Z. 27 (1928), 218-244. 
32. E. M. Stein, Localization and summability of multiple Fourier series, Acta. Math. 100 (1958), 93-147.

33. G. Sonouchi, Discrete analogue of theorem of Littlewood-Paley, Tôhoku Math. J 13 (1961), 295-319.

34. G. Szegö, Asymptotische Entwicklungen der Jacobischen Polynome, Schriften der Königsberger Gelehrten Gesellschaft, naturwissenschaftliche Klasse 10 (1933), 35-112.

35. - Orthogonal polynomials, Amer. Math. Soc. Colloq. Publ. Vol. 23, Amer. Math. Soc., Providence, R. I., 1959.

36. G. M. Wing, The mean convergence of orthogonal series, Amer. J. Math. 72 (1950), 792-807.

37. - On the $L^{p}$ theory of Hankel transforms, Pacific J. Math. 1 (1951), 313-319.

38. A. Zygmund, Trigonometric series, Vols. I and II, Cambridge Univ. Press, Cambridge, 1959.

The University of Wisconsin 